\title{
Cost-Effectiveness of Aspirin Adherence for Secondary Prevention of Cardiovascular Events
}

\author{
Laurence M. Djatche $^{1} \cdot$ Stefan Varga $^{1} \cdot$ Robert D. Lieberthal $^{2}$
}

Published online: 24 April 2018

(C) The Author(s) 2018

\begin{abstract}
Background Suboptimal adherence to aspirin therapy for secondary prevention of cardiovascular $(\mathrm{CV})$ events is an important public health problem. Prior studies have demonstrated non-adherent patients are at higher risk of experiencing $\mathrm{CV}$ events.

Objectives This study aimed to estimate the clinical and economic outcomes of aspirin non-adherence in patients with a prior primary $\mathrm{CV}$ event.

Methods We developed a Markov model to estimate the cost-effectiveness of aspirin adherence from a generic US managed care payer perspective over a 5-year time horizon. Costs, utilities and rates of aspirin adherence, CV events and adverse events were gathered from published literature to populate the model. Outcomes were qualityadjusted life years (QALYs), costs (US\$) and incremental cost-effectiveness ratios (ICERs). We applied the model separately to a population without type II diabetes as a comorbidity (non-diabetic model) and a population with type II diabetes (type II diabetes model). A one-way
\end{abstract}

Robert D. Lieberthal

rliebert@utk.edu

Laurence M. Djatche

laurence.djatche@jefferson.edu

Stefan Varga

svarga89@gmail.com

1 Jefferson College of Population Health, Thomas Jefferson University, 901 Walnut Street, 10th Floor, Philadelphia, PA 19107, USA

2 Department of Public Health, College of Education, Health, and Human Sciences, The University of Tennessee, Knoxville, 1914 Andy Holt Ave., 386 HPER, Knoxville, TN 37996, USA sensitivity analysis was performed to assess the model uncertainty.

Results The base case showed adherent patients lived 0.25 and 0.36 QALYs longer than non-adherent patients in the non-diabetic model and type II diabetes model, respectively. Adherence to aspirin had an ICER of US\$25/QALY in the non-diabetic population, while it saved US\$297 per patient over a 5-year period in the type II diabetes population. One-way sensitivity analysis showed the models were most sensitive to rates of non-fatal events in nonadherent patients.

Conclusion This study suggests aspirin adherence may improve QALYs for patients with a prior primary CV event. Further, it may decrease costs in patients with type II diabetes. While additional research is needed to validate these results, payers may wish to increase strategies to promote adherence in order to improve population health. Trial Registration Not applicable.

\section{Key Points for Decision Makers}

Medication adherence remains a healthcare concern for providers and payers due to the evidence that medication adherence remains suboptimal in cardiovascular (CV) disease patients.

This paper reports the clinical and economic implications of aspirin adherence among $\mathrm{CV}$ disease patients without comorbid diabetes and with type II diabetes.

This paper shows there was a cost decrease only in patients with type II diabetes. 


\section{Introduction}

\subsection{Background}

Cardiovascular (CV) disease is the leading cause of mortality in the United States and represents $17 \%$ of national health expenditures [1]. Patients with a history of a CV event are at higher risk of experiencing a subsequent $\mathrm{CV}$ event if proper pharmacotherapy management is not provided [1-3]. Aspirin is recommended (class I recommendation) by the American Heart Association and the American College of Cardiology Foundation as a preferred antiplatelet for secondary prevention of a CV event [2]. The daily use of aspirin is recommended in coronary artery disease patients unless they present with a contraindication to aspirin $[2,3]$. Generally, patients who follow the recommended medication regimen and take their medications as prescribed at least $80 \%$ of the time are considered "adherent" to a secondary prevention regimen $[4,5]$.

Medication non-adherence for secondary prevention of $\mathrm{CV}$ events is a major concern to providers and payers due to evidence that medication adherence remains suboptimal in CV disease patients [4, 6]. Despite the importance of secondary prevention, a substantial number of patients stop taking their medications soon after being discharged from the hospital [6]. Ho et al. [6] showed that for CV disease patients discharged from the hospital with three preventive medications, about $34 \%$ of all patients stop at least one medication and $12 \%$ of patients stop all medications within 1 month of hospital discharge. For aspirin use, prior research demonstrates that only approximately $71 \%$ of patients with $\mathrm{CV}$ disease are adherent users $[4,5,7]$. The definition of adherence in this study was based on the definitions used in prior adherence studies.

\subsection{Motivation}

Medication adherence is associated with better health outcomes for patients with $\mathrm{CV}$ disease [4, 6, 7]. A number of studies have explored the effects of aspirin adherence as secondary prevention for $\mathrm{CV}$ disease on health outcomes such as myocardial infarction (MI) and stroke $[5,7,8]$. The Reduction of Atherothrombosis for Continued Health (REACH) Registry is an example of a large, international data set that includes both the factors associated with adherence and the consequences of non-adherence over the long term [9].

A few studies have examined the economic implications of aspirin in the US healthcare system and have demonstrated aspirin to be a cost-effective agent for secondary prevention of $\mathrm{CV}$ events compared to other antiplatelet agents [10]. However, a limitation of these studies is the lack of data on the economic consequences of aspirin use for secondary prevention of $\mathrm{CV}$ events in adherent versus non-adherent patients. In general, the economic perspective on medication adherence is still emerging, where classical economic models are not able to fully explain the lack of adherence. Additional studies on the economic implications of adherence may be able to fill this gap in the literature [11].

This study focused on aspirin used as secondary prevention in patients with established CV disease. The objective of this study was to model the impact of adherence to aspirin on healthcare costs, effectiveness [qualityadjusted life years (QALYs)], and cost-effectiveness in patients with a prior primary $\mathrm{CV}$ event, and to apply this model to specific subpopulations for whom secondary prevention is indicated.

\section{Methods}

\subsection{Design and Description}

Two distinct but related Markov models were developed in order to reflect the significant differences in outcomes between $\mathrm{CV}$ disease patients with and without comorbid diabetes. Cost-effectiveness Markov models are used to examine scenarios that involve transitions between various states of health over a period of time when patients can experience multiple possible events [12]. The models in this study were developed and validated through the use of a literature review as described in the model inputs section below (Sect. 2.3) and a prior publication [13]. We also solicited input from a clinical expert in order to develop the models and ensure that they were concordant with current clinical practice and outcomes. Finally, we performed quality assurance checks, including independent verification of the results by an analyst other than the person who implemented the model.

One model represented patients without comorbid diabetes (non-diabetic model) and the other model represented patients with type II diabetes (type II diabetes model). Both types of patients may also have additional comorbidities, such as smoking, high blood pressure, and high cholesterol, but these additional comorbidities are not specifically considered in our modeling approach. All patients entered the model with a prior primary $\mathrm{CV}$ event and were treated with aspirin for secondary prevention of a $\mathrm{CV}$ event. The treatment in this study was adherence to aspirin therapy; outcomes for patients who were non-adherent are a separate branch of our model from adherent patients, and costs and outcomes for those populations were based on the literature on non-adherent populations. In general, we consider that adherence to treatment that achieves greater 
outcomes at lower costs has a relatively higher economic value.

In this model, all patients who had a primary $\mathrm{CV}$ event were considered adherent if they self-reported taking aspirin daily at least $80 \%$ of the time for secondary prevention of $\mathrm{CV}$ events, and non-adherent otherwise [14]. The Markov models were run with a 1-year cycle, meaning that patients were potentially exposed to one event per year. The 1-year cycle was chosen based on the relatively low rates of events in this population and in order to match the 1-year perspective of US managed care payers. There was no time dependency built into the Markov transition probabilities.

Associated QALYs saved were estimated over a 5-year time horizon with one cycle per year. The 5-year time horizon was chosen to allow for the model to calculate outcomes over a longer term horizon, given that $\mathrm{CV}$ disease is a chronic condition. The 5-year time horizon also was designed to appropriately deal with the uncertainty of this disease state, since over longer time horizons, the standard of care and new medical technologies are likely to significantly change the course of treatment. We did not apply a discount rate to costs and QALYs in this study. The model assumed the perspective of a US managed care third-party payer. TreeAge software Pro Core 2016 was used to implement the model [15].

In this model, the probabilities of events for adherent and non-adherent patients were based on a review of the literature, as described below in the "Model Inputs" section; all patients had a chance of experiencing a medical event related to their disease. Events were classified as fatal or non-fatal. Patients who experienced no event as well as non-fatal events reentered the decision tree each cycle, and those who experienced a fatal event exited the decision tree after the fatal event. Non-fatal events included MI, stroke, adverse events related to aspirin and events due to type II diabetes complication. Aspirin adverse events included gastrointestinal (GI) bleeds that resulted in hospitalization. GI bleeds were chosen because they represent among aspirin-related side effects the highest burden on payers and the healthcare system [16]. If patients are not taking aspirin as recommended, they are less likely to experience GI bleeds [17, 18]. As a result, aspirin adverse events were not considered possible clinical outcomes for non-adherent patients in our model.

\subsection{Health Economic Models}

\subsubsection{Non-diabetic Model}

Figure 1 shows the Markov model employed in the analysis. We made the assumption patients in this model do not have type II diabetes and are at a baseline risk of developing a secondary CV event. This model depicted two main arms: adherent and non-adherent to aspirin. This was designed to be concordant with the review of the literature performed to provide model inputs for this study, which utilized "adherent" and "non-adherent" in order to analyze differences between groups, and we followed this practice in the literature. As patients entered the decision tree, they had a one-time choice to be adherent or non-adherent to their medication regimen. All the values of the arms in the non-diabetic model were taken from the literature review as described in the "Model Inputs" section below. The "adherent" and "non-adherent" arms differed in the probabilities of each outcome.

\subsubsection{Type II Diabetes Model}

The structure of the model used in the type II diabetes population was the same as for the non-diabetic model (Fig. 1). This model represents coronary artery disease patients with type II diabetes. The clinical outcomes differed compared to the non-diabetic model due to the higher incidence of events in both the adherent and non-adherent population. The literature review indicated that patients who had diabetes were expected to have peripheral artery disease (PAD) amputations as non-fatal events at costs and rates that will impact payers [19]. We included PAD amputation as a common, long-term and high-cost consequence of inappropriately managed type II diabetes in both the adherent and non-adherent populations.

\subsection{Model Inputs}

All model inputs required to inform the model structure and parameters were based on the findings of a series of literature review studies and peer-reviewed literature reporting on adherence to aspirin and event rates of relevant clinical outcomes. The studies were identified through PubMed search using the keywords "aspirin," "secondary prevention," "coronary artery disease," "myocardial infarction," “stroke," "mortality," "percutaneous coronary intervention," and "adherence." Additionally, previous cost-effectiveness studies on CV disease were reviewed to identify relevant utilities and costs [13].

\subsubsection{Clinical Inputs}

Table 1 contains variables used in the non-diabetic and type II diabetes Markov models. Our assumption was that the types of non-fatal events experienced by adherent and non-adherent patients would be the same, based on the literature on the types of non-fatal events experienced by patients with $\mathrm{CV}$ disease. Adherent patients were assumed to be exposed to GI bleeding as a result of aspirin therapy, 


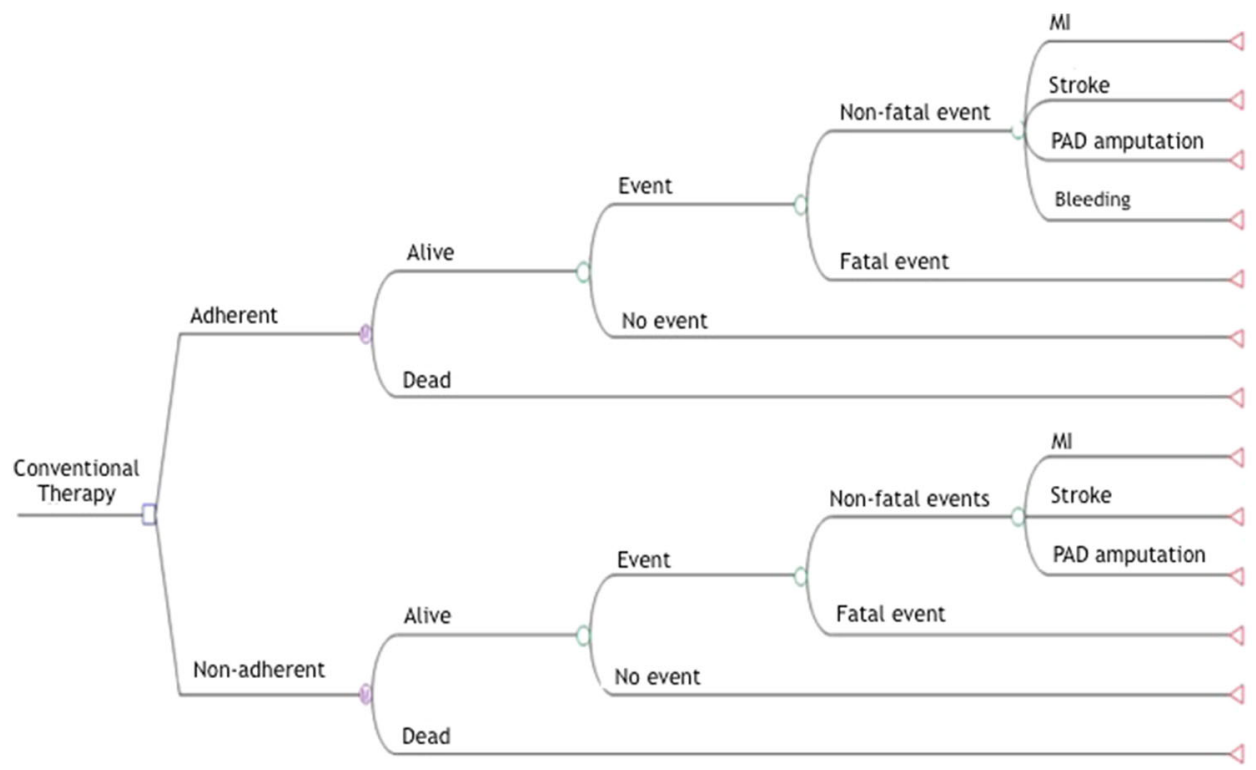

Fig. 1 Cost-effectiveness model structure. Cost-effectiveness model structure comparing aspirin adherence vs aspirin non-adherence for secondary prevention of $\mathrm{CV}$ events. At the decision node ( $\square$ ), patients were assigned as being adherent or non-adherent to aspirin based on the rate found in the literature. Patients entered the decision node with a prior $\mathrm{CV}$ event. In each 1 -year cycle, patients may

while non-adherent patients were not exposed to this nonfatal event. We were able to determine the rates of fatality directly from the literature for $\mathrm{CV}$ disease. The rates of MI and stroke were obtained from a meta-analysis study experience an event (non-fatal or fatal) or no event if they are alive. This model was applied to a population without co-morbidity (nondiabetic model) and a population with type II diabetes (type II diabetes model). $C V$ cardiovascular, GI gastrointestinal, $M I$ myocardial infarction, $P A D$ peripheral arterial disease

comparing aspirin versus placebo [20]. The rate of fatal events was retrieved from a randomized controlled trial [21]. The rate of GI bleeding and PAD amputation were

Table 1 Probability and utilities of events used in the cost-effectiveness model

\begin{tabular}{|c|c|c|c|c|c|c|c|}
\hline \multirow[t]{2}{*}{ Variable } & \multicolumn{3}{|c|}{ Patients without comorbid diabetes } & \multicolumn{3}{|c|}{ Patients with type II diabetes } & \multirow[t]{2}{*}{ Sensitivity analysis range tested } \\
\hline & Adherent & Non-adherent & References & Adherent & Non-adherent & References & \\
\hline Event $^{\mathrm{a}}$ & 0.158 & 0.174 & Calculated & 0.395 & 0.464 & Calculated & $50-200 \%$ of base case \\
\hline Non-fatal event ${ }^{b}$ & 0.035 & 0.026 & Calculated & 0.069 & 0.070 & Calculated & $50-200 \%$ of base case \\
\hline MI & 0.008 & 0.012 & {$[20]$} & 0.022 & 0.032 & {$[20]$} & \\
\hline Stroke & 0.011 & 0.014 & {$[20]$} & 0.029 & 0.036 & {$[20]$} & \\
\hline PAD amputation & - & - & Assumption & 0.002 & 0.002 & {$[22]$} & \\
\hline GI bleeding & 0.016 & - & [18] & 0.016 & - & {$[18]$} & \\
\hline Fatal events & 0.123 & 0.148 & {$[21]$} & 0.326 & 0.394 & {$[21]$} & \\
\hline No event ${ }^{c}$ & 0.842 & 0.826 & Calculated & 0.605 & 0.536 & Calculated & \\
\hline Utilities & & & [27-29] & & & {$[27-29]$} & $50-110 \%$ of base case \\
\hline MI & 0.850 & 0.850 & & 0.850 & 0.850 & & \\
\hline Stroke & 0.650 & 0.650 & & 0.650 & 0.650 & & \\
\hline PAD amputation & - & - & & 0.610 & 0.610 & & \\
\hline GI bleeding & 0.800 & 0.800 & & 0.800 & 0.800 & & \\
\hline
\end{tabular}

$G I$ gastrointestinal, $M I$ myocardial infarction, $P A D$ peripheral arterial disease

${ }^{\mathrm{a}}$ Rate of events is the sum of non-fatal events and fatal events

${ }^{\mathrm{b}}$ Rate of non-fatal events is the sum of the rate of MI, stroke, PAD amputation and GI bleeding

${ }^{\mathrm{c}}$ Rate of no events is the rate of events subtracted from 1 
respectively retrieved from a retrospective cohort study and cross sectional study [18, 22].

The total rate of events in the adherent and non-adherent populations was calculated by adding the fatal and nonfatal event rates found in the literature [18, 20-22]. The rates of events were subtracted from 1 to determine the rates of no events (Table 1). To determine the rates of events in the type II diabetes model, a hazard ratio of 2.66 was taken from a meta-analysis study of primary prevention showing an increased rate of MI, stroke, or fatal events in a diabetic population [20]. Due to the lack of data on secondary prevention, we applied this hazard ratio in our model under the assumption that the secondary prevention event rates would be similar, or worse, than in a primary prevention setting.

\subsubsection{Cost Variables}

Cost variables were collected from a number of sources and are shown in Table 2. Costs for events were available in aggregate and were not broken down by component. The cost of aspirin was not included in the model because it is low and it would not affect the overall cost of treatment from a managed care payer perspective [17]. The costs of fatal events, non-fatal MI, non-fatal stroke and GI bleeds were obtained from previous cost-effectiveness studies $[23,24]$. Due to a lack of data in the literature, we used the cost of fatal congestive heart failure as the cost of fatal events despite the fact that fatal MI or fatal stroke are more likely to be fatal events for patients who had a primary CV event. The cost of PAD amputation caused by uncontrolled diabetes was gathered from an Agency for Healthcare Research and Quality (AHRQ) report on the economic burden of diabetes [25]. All expenditures were inflated to January 2016 US dollars using the medical care component of the US consumer price index (CPI-U) by multiplying by the value of the Consumer Price Index for All Urban Consumers: Medical Care (CPI-U Medical Care) in January 2016 and dividing by the value at the time that the cost data were collected and reported [26].

\subsubsection{Utilities}

Health state utilities were obtained from published literature that used standardized methods for patient-reported outcomes [27-29]. Utilities of MI and stroke were obtained from previous cost-effectiveness studies using EQ-5D scores in the US MI FREEE trial [27]. EQ-5D scores collected from diabetes patients with type I and type II who had been treated for foot ulcers by a multidisciplinary team at Lund University Hospital in Sweden were used to populate the utility after PAD-related amputation [28]. Utility of GI hemorrhage obtained from a previous cost-effectiveness study in the UK using time trade-off score and a prior outcomes research trial in the US was used to populate the utility of patients who experienced GI bleeding [29-31].

\subsection{Model Outputs}

For the base-case analysis, the models' endpoints included the expected outcomes of both costs and QALYs, resulting in a cost-effectiveness ratio. We followed the standard method of calculating the incremental (marginal) costs per QALY for adherent and non-adherent patients by calculating an incremental cost-effectiveness ratio (ICER) [12]. The ICER compared the relative cost-effectiveness of aspirin adherence and aspirin non-adherence in patients without comorbid diabetes and with type II diabetes. In the case of this study, non-adherent strategy was set as the reference compared to the adherent strategy. ICER was not calculated if a strategy was either dominant or dominated between the two strategies. A strategy was dominant if it achieved better outcomes (QALYs) at lower costs compared to the other strategy.

\subsection{Sensitivity Analysis}

A sensitivity analysis was performed to examine the robustness of the results obtained from the health economic models. One-way sensitivity analysis was performed with a wide range of percentage to identify the influential

Table 2 Cost variables from the literature used in the cost effectiveness model

\begin{tabular}{llllll}
\hline Event & Cost per event in the literature & Year reported in the literature & 2016 cost & References & Base-case sensitivity analysis \\
\hline MI & US $\$ 16,563$ & 2006 & US $\$ 22,119$ & {$[23]$} & $50-200 \%$ of base case \\
Stroke & US $\$ 13,878$ & 2006 & US $\$ 18,533$ & {$[23]$} & $50-200 \%$ of base case \\
PAD amputation & US $\$ 7700$ & 2006 & US $\$ 10,283$ & {$[25]$} & $50-200 \%$ of base case \\
Fatal CHF & US $\$ 8782$ & 2006 & US $\$ 11,728$ & {$[23]$} & $50-200 \%$ of base case \\
GI bleeding & US $\$ 6866$ & 1989 & US $\$ 20,489$ & {$[24]$} & $50-200 \%$ of base case \\
\hline
\end{tabular}

$C H F$ congestive heart failure, $G I$ gastrointestinal, $M I$ myocardial infarction, $P A D$ peripheral arterial disease 
parameters on the base-case analysis. Changes in probability of events, probability of non-fatal events, costs and utilities were performed to assess the degree to which the model parameter estimates affected our results in both the non-diabetic model and the type II diabetes model (see Tables 1 and 2). Due to the variety of published literature used in the model and the lack of information on $95 \%$ confidence intervals, we used $50-200 \%$ of the base-case estimates of all parameters, except utilities. Utilities were varied from 50 to $110 \%$ to avoid exceeding the maximum utility value of 1 . A tornado diagram was used to determine the parameters that most influenced our models' results considering the ICER.

\section{Results}

\subsection{Base-Case Analysis}

Cumulative survival and cumulative costs over the 5-year period of the model were used to calculate total and incremental outcomes. The average 5-year survival among patients without comorbid diabetes was 0.25 years higher in the adherent population. In the non-diabetic model, patients who were adherent to aspirin had a cumulative cost of US\$8420, while the cost of non-adherent patients was US\$8414. Note that this cost difference does not include the cost of aspirin therapy for adherent patients on the assumption that aspirin is purchased as an over-the-counter medication without the use of insurance benefits. The cost per QALY was US\$2671 among the adherent population and US\$2476 among the non-adherent population. As a result, there was no dominant strategy in the non-diabetic model, and an ICER was calculated. The ICER showed for every US\$25 spent in the adherent population, one QALY is saved (Table 3).

In the type II diabetes model, the average 5-year survival was 0.36 years higher in the adherent population. Patients who were adherent to aspirin had a cumulative cost of US $\$ 13,727$, while the cost for non-adherent patients was US\$14,024. The cost per QALY was US\$7934 among the adherent population and US\$10,237 among the nonadherent population, representing a US\$2303 difference in this metric. As a result, adherence had a cost-saving economic outcome in addition to improved clinical outcomes in the type II diabetes population, making it the dominant strategy.

\subsection{Sensitivity Analysis}

The univariate analysis showed our model is sensitive to variation of certain parameters compared to others. Overall, the model results were more sensitive to changes of parameters in the non-diabetic model compared to the model with type II diabetes patients. In both models, tornado diagrams showed ICER results were highly sensitive to variation of the rate of non-fatal events in non-adherent patients. Those events include MI, stroke, GI bleeding and PAD amputations, meaning we had to be precise in our estimates for those probabilities. The models were not sensitive to varying costs and utilities over a range of values (see Fig. 2).

\section{Discussion}

\subsection{Potential Explanations for the Results}

Based on the results of our base case, there are possible explanations for the cost and QALY outcomes obtained from the two models. From the point of view of survival, the models showed a benefit of adherence over non-adherence consistent with our review of the literature [7, 8]. The difference in survival is driven by the higher probability of a fatal event in the non-adherent population. The differences in survival are not only based on the higher rates of fatal events, but they are based on the setup of the Markov model. Patients who begin the model as adherent remained adherent for all 5 years, and those who begin the model as non-adherent remained non-adherent for all 5 years. In general, non-adherence has been identified as a potential cause of higher costs in the healthcare system, and prior studies of the post-MI population have identified full drug coverage as an intervention that can save both lives and money $[4,23]$.

In the non-diabetic model, patients who were adherent to aspirin had a higher cumulative cost compared to patients who were non-adherent to aspirin. However, in the type II diabetes model, the cumulative cost of aspirin adherence was slightly lower compared to the cost of aspirin non-adherence. The increased cost of the type II diabetes population when compared to the non-diabetic population exists despite the fact that the non-diabetic population lives longer, and thus has a longer period of exposure to healthcare events. The higher cost of the type II diabetes population is driven primarily by the much higher rate of CV-related events. The higher cost of diabetes-related events represented in the model by PAD amputation has a relatively small contribution due to its relatively low event rate and direct costs. Finally, the study model only tested outcomes over a 5-year horizon, meaning that our results may differ if the model was simulated over a longer time horizon. 
Table 3 Cost-effectiveness results of aspirin adherence for the base case in the non-diabetic population and type II diabetes population

\begin{tabular}{llllll}
\hline Population & Strategy & $\begin{array}{l}\text { Average 5-year cost } \\
\text { per member }\end{array}$ & $\begin{array}{l}\text { Average 5-year } \\
\text { survival (QALYs) }\end{array}$ & $\begin{array}{l}\text { Cost-effectiveness } \\
\text { (cost per QALY) }\end{array}$ & $\begin{array}{l}\text { Incremental cost-effectiveness } \\
\text { ratio (cost per QALY) }\end{array}$ \\
\hline $\begin{array}{l}\text { Patients without } \\
\text { comorbid diabetes }\end{array}$ & $\begin{array}{c}\text { Non- } \\
\text { adherent } \\
\text { Adherent }\end{array}$ & US\$8414 & 3.15 & US\$2671 & Reference \\
$\begin{array}{c}\text { Patients with type II } \\
\text { diabetes }\end{array}$ & $\begin{array}{c}\text { Non- } \\
\text { adherent }\end{array}$ & US $\$ 14,024$ & 3.40 & US $\$ 2476$ & US\$25 \\
& Adherent & US $\$ 13,727$ & 1.37 & US $\$ 10,237$ & Reference \\
\hline
\end{tabular}

$Q A L Y$ quality-adjusted life year

Fig. 2 One-way sensitivity analyses tornado diagram for aspirin adherence vs aspirin non-adherence. The horizontal bars show the effect varying each variable over a range of values has on the ICER. The larger the horizontal bar, the more sensitive the model is to the varying parameter.

a Tornado diagram evaluating the influence of each parameter of the non-diabetic model on ICER. b Tornado diagram evaluating the influence of each parameter of the type II diabetes model on ICER. GI gastrointestinal, ICER incremental cost-effectiveness ratio, $M I$ myocardial infarction, $P A D$ peripheral arterial disease

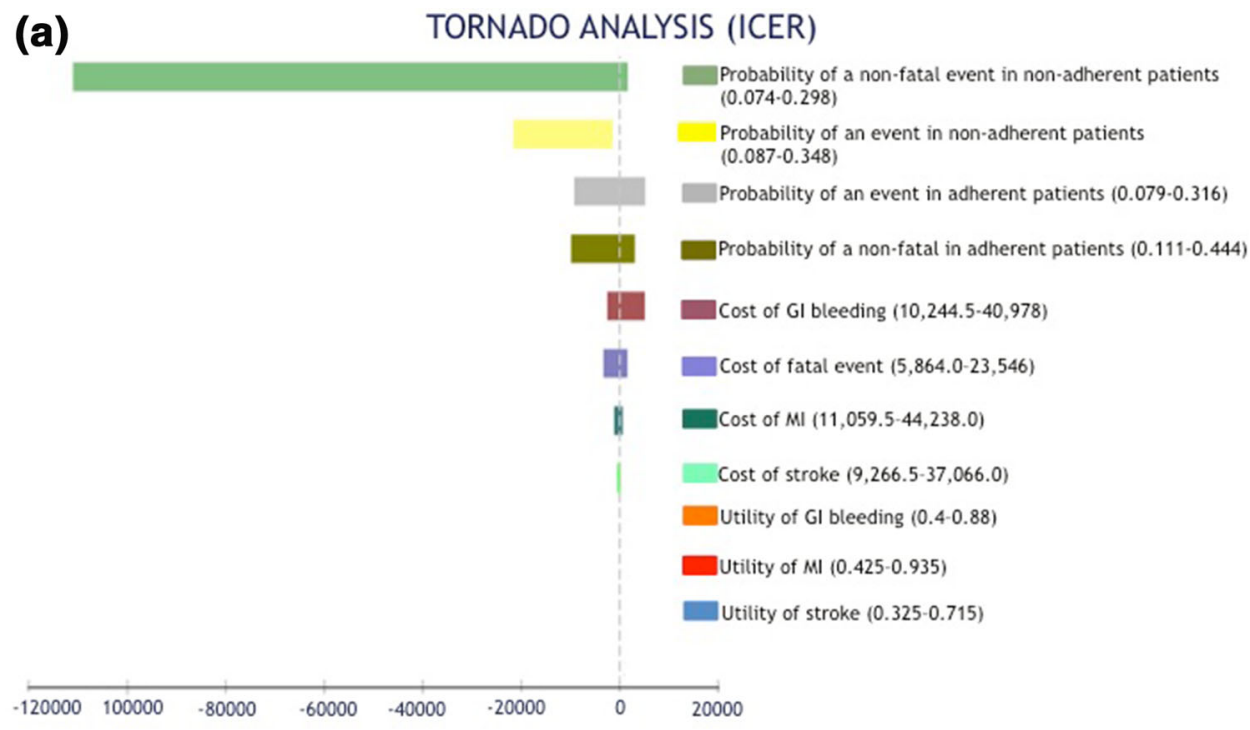

(b) TORNADO ANALYSIS (ICER)

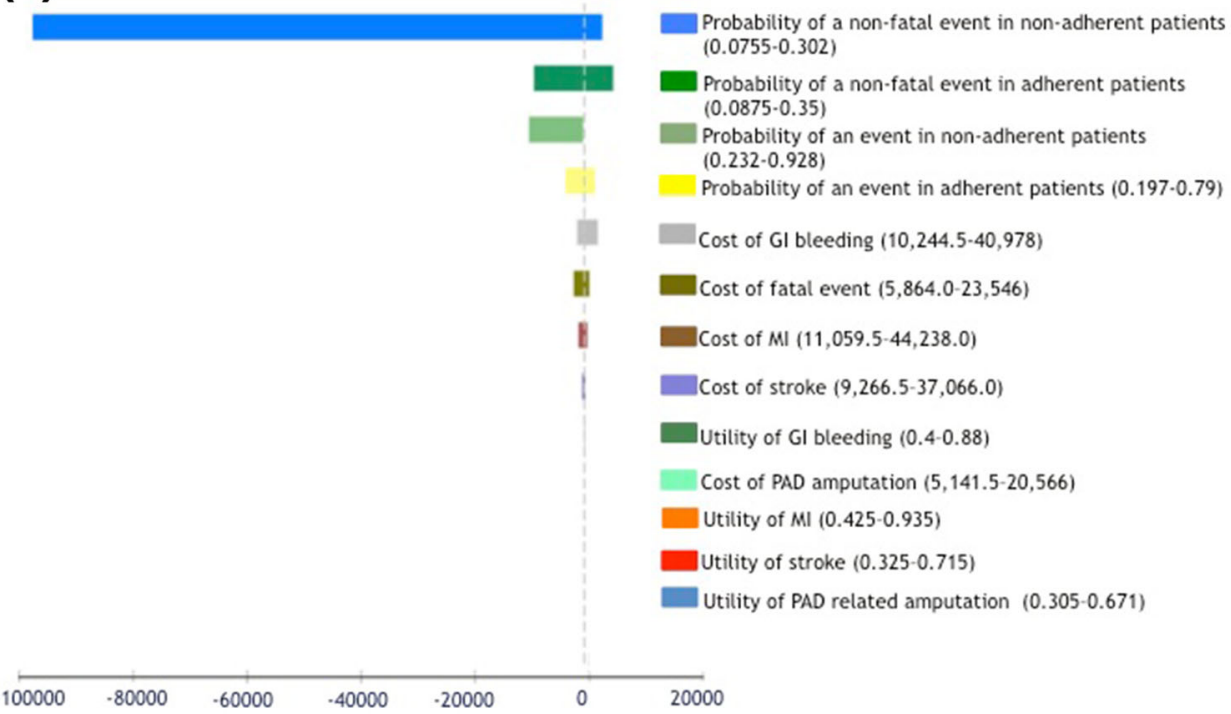




\subsection{Limitations}

In considering our results, it is important to note that multiple sources were used to obtain the model inputs, including probabilities, costs and utilities. The one-way sensitivity analysis shows our model is sensitive to varying rates of non-fatal events in adherent and non-adherent patients. This is explained by different patient-related factors, such as demographics or medical history, that vary across the studies used. It may affect the rates of events obtained in one study compared to another one. If our findings are correct, future randomized controlled trials to promote adherence should be designed and powered to detect such a difference. Randomized controlled trials would be the ideal way to test interventions designed to promote adherence based both on our results and the limitations of the modeling approach to the research question. From a research point of view, randomized controlled trials would both control for confounding and collect participantspecific cost and outcomes data.

There are other important limitations to our study. First, patients can experience more than one CV event within 1 year. We made the assumptions patients can experience one event per year, which may vary based on a patient's risk factors. Also, this study was done with a 5-year time horizon, and the rates of experiencing a third and fourth $\mathrm{CV}$ event might differ from the probability of experiencing a second event. The same probability of developing a second event was used in all the cycles due to the set-up of the Markov model.

Another limitation is the inconsistent definition of adherence; medication adherence is defined and measured differently across the literature. There is no standard measure of adherence, and some studies used multiple methods or thresholds to assess adherence [32, 33]. In our study, adherence inputs were obtained from patients who self-reported taking aspirin at least $80 \%$ of the time after discharge from the hospital. Studies using self-report present an additional barrier, due to the potential for intentional or unintentional provision of inaccurate information $[32,34]$.

The relatively strong assumption that adherent patients are receiving treatment, while those who are non-adherent are in some sense "untreated" is also a limitation of our study. Adherence may be measured through the use of a binary variable; however, adherence is a dynamic measurement; it is a gradient (not binary) and can change over time. This is especially true over a 5-year window. In addition, non-adherent patients with a prior CV event are likely receiving a substantial amount of treatment on average, especially if they have co-morbid type II diabetes. The extent to which this assumption biases our results depends crucially on whether adherent individuals also tend to receive more treatment, or if non-adherent individuals compensate by seeking other medical or drug therapy. This is a key point that should be addressed in future studies.

Finally, costs captured from the literature might miss important cost components. The costs used in our model were obtained from different sources and were from 2006 and 1989, which may not fully reflect the cost of today's practice. This is true for the components that make up the total cost of each event in the model and for the differences in price and utilization that are not adequately reflected in our use of the inflation index factor. Managed care payers may find it more useful to utilize their own data, especially for the cost of GI bleeding, and to rerun the model, which we have shared, as described in the "Data Availability Statement" section of this paper. In addition, the one-way sensitivity analysis shows that our model is not sensitive to the costs of those events.

\subsection{Conclusions}

This economic model was developed to investigate health benefits and economic implications that could be expected from adherence to aspirin for secondary prevention of a $\mathrm{CV}$ event. Our approach in this study is likely to be applicable to other studies in this area because we defined two population characteristics and applied the general model to each population. This facilitates our understanding about the impact of adherence on health outcomes and costs in both populations. It also makes our model more generalizable to a specific type of population, patients with type II diabetes.

We found that adherence to aspirin has the potential to improve health outcomes and save money in patients with type II diabetes for third-party payers. Within a 5-year time horizon, our results showed that adherence provided a US\$297 cost-saving per patient with type II diabetes, and an ICER of US\$25 per QALY in patients without comorbid diabetes. This model informs third-party payers for decision making regarding adherent and non-adherent $\mathrm{CV}$ patients who are taking guideline-recommended medications such as aspirin. It also informs the potential value of interventions that could raise the rates of adherence in an insured population.

In conclusion, this analysis suggests that adherence to aspirin increases QALYs and reduces cost in patients with a prior CV event, but only for patients with type II diabetes. Given our findings, managed care organizations, government payers and patients should consider participating in programs that improve medication adherence, as long as intervention costs do not exceed the estimated healthcare savings. While additional research is needed to validate those results in the real world, it is important to develop 
strategies that will increase adherence and improve population health.

Acknowledgements The model used in this study was provided to the journal's peer reviewers for their reference when reviewing the manuscript. Dr. David Whellan provided clinical expertise used to validate that the models reflected current practice, as part of a prior study [13].

Authors contributions Dr. Varga was primarily responsible for developing the health economic model used in this study. Dr. Djatche was primarily responsible for inputting QALYs into this model, running the sensitivity analysis, and writing the manuscript. Dr. Lieberthal was responsible for designing the study, including the research question, overseeing the scientific work for the study, and providing quality assurance for the results. Dr. Lieberthal will act as the overall guarantor for this work.

Data Availability Statement The authors are making the data for this study open and available. All data used in the analyses are available in the article tables. The TreeAge models described in this study are available in the University of Tennessee's Tennessee Research and Creative Exchange (Trace) system at http://trace. tennessee.edu/utk_publichealth/4/. Please note that this link will allow users to download both the non-diabetes and type II diabetes models as TreeAge files (.trex).

\section{Compliance with Ethical Standards}

This work was previously presented at the 2017 International Society for Pharmacoeconomics and Outcomes Research (ISPOR) meeting in Boston, MA. The abstract was published in the journal Value in Health [35].

Funding No funding was received for this study.

Conflict of interest Dr. Varga and Dr. Djatche's efforts were supported by a fellowship funded by Novartis, and both were employees at Jefferson at the time the study was done. Dr. Lieberthal reports previously receiving funding from Novartis as a mentor for the university fellows program and research funding from New Haven Pharmaceuticals. All such funds were paid to Thomas Jefferson University. He also has received consulting fees from Shire Pharmaceuticals for consulting unrelated to aspirin therapy or cardiovascular disease.

Open Access This article is distributed under the terms of the Creative Commons Attribution-NonCommercial 4.0 International License (http://creativecommons.org/licenses/by-nc/4.0/), which permits any noncommercial use, distribution, and reproduction in any medium, provided you give appropriate credit to the original author(s) and the source, provide a link to the Creative Commons license, and indicate if changes were made.

\section{References}

1. Heidenreich PA, Trogdon JG, Khavjou OA, et al. Forecasting the future of cardiovascular disease in the united states: a policy statement from the American Heart Association. Circulation. 2011;123(8):933-44. https://doi.org/10.1161/CIR.0b013e31820a $55 f 5$.

2. Smith SC Jr, Benjamin EJ, Bonow RO, et al. AHA/ACCF secondary prevention and risk reduction therapy for patients with coronary and other atherosclerotic vascular disease: 2011 update: a guideline from the American Heart Association and American College of Cardiology foundation. Circulation. 2011;124(22): 2458-73. https://doi.org/10.1161/CIR.0b013e318235eb4d.

3. US Preventive Services Task Force. Aspirin for the prevention of cardiovascular disease: U.S. preventive services task force recommendation statement. Ann Intern Med. 2009;150(6):396-404 (150/6/396).

4. Osterberg L, Blaschke T. Adherence to medication. N Engl J Med. 2005;353(5):487-97 (353/5/487 [pii]).

5. Ho PM, Magid DJ, Shetterly SM, et al. Importance of therapy intensification and medication nonadherence for blood pressure control in patients with coronary disease. Arch Intern Med. 2008;168(3):271-6. https://doi.org/10.1001/archinternmed.2007. 72.

6. Ho PM, Bryson CL, Rumsfeld JS. Medication adherence: its importance in cardiovascular outcomes. Circulation. 2009;119(23): 3028-35. https://doi.org/10.1161/CIRCULATIONAHA.108. 768986.

7. Amin AP, Mukhopadhyay E, Nathan S, Napan S, Kelly RF. Association of medical noncompliance and long-term adverse outcomes, after myocardial infarction in a minority and uninsured population. Transl Res. 2009;154(2):78-89. https://doi.org/10. 1016/j.trsl.2009.05.004.

8. Biondi-Zoccai GG, Lotrionte M, Agostoni P, et al. A systematic review and meta-analysis on the hazards of discontinuing or not adhering to aspirin among 50,279 patients at risk for coronary artery disease. Eur Heart J. 2006;27(22):2667-74 (ehl334 [pii]).

9. Rodriguez F, Cannon CP, Steg PG, Kumbhani DJ, Goto S, Smith SC, Eagle KA, Ohman EM, Umez-Eronini AA, Hoffman E. Bhatt DL; REACH Registry Investigators. Predictors of long-term adherence to evidence-based cardiovascular disease medications in outpatients with stable atherothrombotic disease: findings from the REACH Registry. Clin Cardiol. 2013;36(12):721-7. https:// doi.org/10.1002/clc.22217.

10. Gaspoz JM, Coxson PG, Goldman PA, et al. Cost effectiveness of aspirin, clopidogrel, or both for secondary prevention of coronary heart disease. N Engl J Med. 2002;346(23):1800-6. https://doi. org/10.1056/NEJM200206063462309.

11. Elliott RA, Shinogle JA, Peele P, Bhosle M, Hughes DA. Understanding medication compliance and persistence from an economics perspective. Value Health. 2008;11(4):600-10. https:// doi.org/10.1111/j.1524-4733.2007.00304.x.

12. Drummond M, editor. Methods for the economic evaluation of health care programmes. 3rd ed. New York: Oxford University Press; 2005.

13. Lieberthal RD, Varga S, Adams S, McCarey M, Toroni B, Whellan D. The economic implications of non-adherence to aspirin in patients with prior primary cardiovascular events. Circulation. 2016;134(suppl 1):A13150.

14. Shore S, Jones PG, Maddox TM, et al. Longitudinal persistence with secondary prevention therapies related to patient risk after myocardial infarction. Heart. 2015;101(10):800-7.

15. TreeAge Pro 2016 (version release R1.1). TreeAge software, inc. www.treeage.com Web site. www.treeage.com. Updated 2016. Accessed 21 Nov 2015.

16. Manson SC, Benedict A, Pan F, Wittrup-Jensen KU, Fendrick AM. Potential economic impact of increasing low dose aspirin usage on CVD in the US. Curr Med Res Opin. 2010;26(10):2365-73. https://doi.org/10.1185/03007995.2010. 514481.

17. Micromedex Solutions. Aspirin. Truven Health Analytics, Inc Web site. http://www.micromedexsolutions.com. Updated 2016. Accessed 24 July 2016.

18. Hedberg J, Sundstrom J, Thuresson M, Aarskog P, Oldgren J, Bodegard J. Low-dose acetylsalicylic acid and gastrointestinal 
ulcers or bleeding-a cohort study of the effects of proton pump inhibitor use patterns. J Intern Med. 2013;274(4):371-80. https:// doi.org/10.1111/joim.12103.

19. Pelletier EM, Smith PJ, Boye KS, Misurski DA, Tunis SL, Minshall ME. Direct medical costs for type 2 diabetes mellitus complications in the US commercial payer setting: a resource for economic research. Appl Health Econ Health Policy. 2008;6(2-3):103-12. https://doi.org/10.2165/00148365200806020-00003.

20. Baigent C, Blackwell L, Antithrombotic Trialists' (ATT) Collaboration, et al. Aspirin in the primary and secondary prevention of vascular disease: collaborative meta-analysis of individual participant data from randomised trials. Lancet. 2009;373(9678):1849-60. https://doi.org/10.1016/s01406736(09)60503-1.

21. Elwood PC, Sweetnam PM. Aspirin and secondary mortality after myocardial infarction. Lancet. 1979;2(8156-8157):1313-5 (S0140-6736(79)92808-3 [pii]).

22. Goodney PP, Holman K, Henke PK, et al. Regional intensity of vascular care and lower extremity amputation rates. J Vasc Surg. 2013;57(6):1471-9. https://doi.org/10.1016/j.jvs.2012.11.068 (1480.e1-3; discussion 1479-80).

23. Choudhry NK, Patrick AR, Antman EM, Avorn J, Shrank WH. Cost-effectiveness of providing full drug coverage to increase medication adherence in post-myocardial infarction medicare beneficiaries. Circulation. 2008;117(10):1261-8. https://doi.org/ 10.1161/CIRCULATIONAHA.107.735605.

24. Edelson JT, Tosteson AN, Sax P. Cost-effectiveness of misoprostol for prophylaxis against nonsteroidal anti-inflammatory drug-induced gastrointestinal tract bleeding. JAMA. 1990;264(1):41-7.

25. Margolis DJ, Malay DS, Hoffstad OJ, et al. Economic burden of diabetic foot ulcers and amputations: Data points \#3. In: Data points publication series. Rockville (MD). 2011. NBK65152 [bookaccession].

26. US Bureau of Labor Statistics Data. Consumer price indexmedical component- CPI inflation calculator. Bureau of Labor
Statistics Web site. https://www.bls.gov/data/inflation_calculator. htm. Updated 2017. Accessed 24 July 2016.

27. Ito K, Avorn J, Shrank WH, et al. Long-term cost-effectiveness of providing full coverage for preventive medications after myocardial infarction. Circ Cardiovasc Qual Outcomes. 2015;8(3):252-9. https://doi.org/10.1161/CIRCOUTCOMES.114.001330.

28. Ragnarson Tennvall G, Apelqvist J. Health-related quality of life in patients with diabetes mellitus and foot ulcers. J Diabetes Compl. 2000;14(5):235-41 (S1056-8727(00)00133-1 [pii]).

29. Gerson L, Kamal A. Cost-effectiveness analysis of management strategies for obscure GI bleeding. Gastrointest Endosc. 2008;68(5):920-36. https://doi.org/10.1016/j.gie.2008.01.035.

30. Thomson R, Parkin D, Eccles M, Sudlow M, Robinson A. Decision analysis and guidelines for anticoagulant therapy to prevent stroke in patients with atrial fibrillation. Lancet. 2000;355(9208):956-62.

31. Fryback DG, Dasbach EJ, Klein R, Klein BE, Dorn N, Peterson K, Martin PA. The Beaver Dam Health Outcomes Study: initial catalog of health-state quality factors. Med Decis Making. 1993;13(2):89-102.

32. Lansberg MG, O'Donnell MJ, Khatri P, et al. Antithrombotic and thrombolytic therapy for ischemic stroke: antithrombotic therapy and prevention of thrombosis, 9th ed: American college of chest physicians evidence-based clinical practice guidelines. Chest. 2012;141(2 Suppl):e601S-36S (S0012-3692(12)60133-0 [pii]).

33. Naderi SH, Bestwick JP, Wald DS. Adherence to drugs that prevent cardiovascular disease: meta-analysis on 376,162 patients. Am J Med. 2012;125(9):882.e1-887.e1. https://doi.org/ 10.1016/j.amjmed.2011.12.013.

34. Monnette A, Zhang Y, Shao H, Shi L. Concordance of adherence measurement using self-reported adherence questionnaires and medication monitoring devices: an updated review. Pharmacoeconomics. 2018;36(1):17-27. https://doi.org/10.1007/s40273017-0570-9.

35. Djatche L, Varga S, Liebertal R. Long term cost effectiveness of adherence to aspirin for secondary prevention of cardiovascular events. Value Health. 2017;20(5):A9. 